\title{
Spontaneous symmetry breaking in a quenched ferromagnetic spinor Bose condensate
}

\author{
L. E. Sadler, J. M. Higbie, S. R. Leslie, M. Vengalattore and D. M. Stamper-Kurn \\ Department of Physics, University of California, Berkeley CA 94720
}

A central goal in condensed matter and modern atomic physics is the exploration of manybody quantum phases and the universal characteristics of quantum phase transitions in so far as they differ from those established for thermal phase transitions. Compared with condensedmatter systems, atomic gases are more precisely constructed and also provide the unique opportunity to explore quantum dynamics far from equilibrium. Here we identify a second-order quantum phase transition in a gaseous spinor BoseEinstein condensate, a quantum fluid in which superfluidity and magnetism, both associated with symmetry breaking, are simultaneously realized. ${ }^{87} \mathrm{Rb}$ spinor condensates were rapidly quenched across this transition to a ferromagnetic state and probed using in-situ magnetization imaging to observe spontaneous symmetry breaking through the formation of spin textures, ferromagnetic domains and domain walls. The observation of topological defects produced by this symmetry breaking, identified as polar-core spin-vortices containing non-zero spin current but no net mass current, represents the first phase-sensitive in-situ detection of vortices in a gaseous superfluid.

Most ultracold atomic gases consist of atoms with nonzero total angular momentum denoted by the quantum number $F$, which is the sum of the total electronic angular momentum and nuclear spin. In spinor atomic gases, such as $F=1$ and $F=2$ gases of ${ }^{23} \mathrm{Na}\left[1,[2]\right.$ and ${ }^{87} \mathrm{Rb}$ [3, 4], all magnetic sublevels representing all orientations of the atomic spin may be realized [5]. The phase coherent portion of a Bose-Einsein condensed spinor gas is described by a vector order parameter and therefore exhibits spontaneous magnetic ordering. Nevertheless, considerable freedom remains for the type of ordering that can occur. For ${ }^{87} \mathrm{Rb} F=1$ spinor gases, the spindependent energy per particle in the condensate is the sum of two terms, $c_{2} n\langle\overrightarrow{\hat{F}}\rangle^{2}+q\left\langle\hat{F}_{z}^{2}\right\rangle$, where $\overrightarrow{\hat{F}}$ denotes the dimensionless spin vector operator. The first term describes spin-dependent interatomic interactions, with $n$ being the number density and $c_{2}=\left(4 \pi \hbar^{2} / 3 m\right)\left(a_{2}-a_{0}\right)$ depending on the atomic mass $m$ and the $s$-wave scattering lengths $a_{f}$ for collisions between pairs of particles with total spin $f[6,6]$. Given $c_{2}<0$ for our system $[3,4,8,[9]$, the interaction term alone favors a ferromagnetic phase with broken rotational symmetry. The second term describes a quadratic Zeeman shift in our experiment, with $q=\left(h \times 70 \mathrm{~Hz} / \mathrm{G}^{2}\right) B^{2}$ at a magnetic field of magnitude $B$ [10]. This term favors instead a scalar phase with no net magnetization, i.e. a condensate in the $\left|m_{z}=0\right\rangle$ magnetic sublevel. These phases are divided by a second-order quantum phase transition at $q=2\left|c_{2}\right| n$.

This article describes our observation of spontaneous symmetry breaking in a ${ }^{87} \mathrm{Rb}$ spinor BEC that is rapidly quenched across this quantum phase transition. Nearlypure spinor Bose-Einstein condensates were prepared in the scalar $\left|m_{z}=0\right\rangle$ phase at a high quadratic Zeeman shift $\left(q \gg 2\left|c_{2}\right| n\right)$. By rapidly reducing the magnitude of the applied magnetic field, we quenched the system to conditions in which the ferromagnetic phase is energetically favored $\left(q \ll 2\left|c_{2}\right| n\right)$. At variable times $T_{\text {hold }}$ after the quench, high-resolution maps of the magnetization vector density were obtained using magnetizationsensitive phase contrast imaging [11]. Soon after the quench, transverse ferromagnetic domains of variable size formed spontaneously throughout the condensate, divided by narrow unmagnetized domain walls separating domains of nearly opposite orientation. Concurrent with the formation of these domains, we also observed topological defects that we characterize as single charged spin vortices with non-zero circulation of spin current and an unmagnetized filled core.

Spinor BECs in the $\left|F=1, m_{z}=0\right\rangle$ hyperfine state were confined in an optical dipole trap characterized by oscillation frequencies $\left(\omega_{x}, \omega_{y}, \omega_{z}\right)=2 \pi(56,350,4.3)$ $\mathrm{s}^{-1}$. The condensates, typically containing $2.1(1) \times 10^{6}$ atoms, were formed at a magnetic field of $2 \mathrm{G}$ and were characterized by a peak density $n_{0}=2.8 \times 10^{14} \mathrm{~cm}^{-3}$ and Thomas-Fermi radii $\left(r_{x}, r_{y}, r_{z}\right)=(12.8,2.0,167)$ $\mu \mathrm{m}$ (Supplementary Note 1). The anisotropic condensates were effectively two-dimensional with respect to the spatial variations in the internal-state wavefunction due to the fact that the spin healing length, $\xi_{s}=$ $\sqrt{\hbar^{2} / 2 m\left|c_{2}\right| n_{0}}=2.4 \mu \mathrm{m}$, is larger than $r_{y}$, therfore eliminating spin dynamics in the $\hat{y}$ direction. Thus, imaging the condensate in the $\hat{x}-\hat{z}$ plane produces complete maps of the magnetization density. The reduced dimensionality also determines the nature of topological defects that may arise in this quantum fluid.

After the condensate was formed, the magnetic field was ramped linearly over $5 \mathrm{~ms}$ to a magnitude of $50 \mathrm{mG}$ in the $\hat{z}$ direction and was held at this setting for a variable time $T_{\text {hold }}$ prior to the imaging sequence. At this field, the quadratic Zeeman energy $q=h \times 0.2 \mathrm{~Hz}$ is negligible compared to the spin-dependent interaction energy of (4/5)2 $\left|c_{2}\right| n_{0}=h \times 8.2 \mathrm{~Hz}$, which is computed using the density averaged in the $\hat{y}$ direction.

The condensate magnetization was measured in-situ using phase-contrast imaging with pulses of circular polarized light, yielding an optical signal given approximately as $\zeta\left(1+\frac{5}{6}\left\langle\hat{F}_{y}\right\rangle+\frac{1}{6}\left\langle\hat{F}_{y}^{2}\right\rangle\right)$ 11]. Here, $\zeta=\tilde{n} \sigma \gamma / 8 \delta$ 


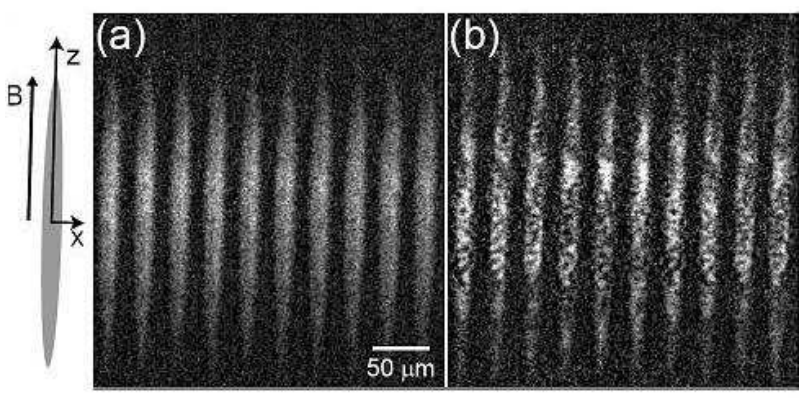

FIG. 1: Direct imaging of inhomogeneous spontaneous magnetization of a spinor BEC. Transverse imaging sequences (first 10 of 24 frames taken) are shown (a) for a single condensate probed at $T_{\text {hold }}=36 \mathrm{~ms}$ and (b) for a different condensate at $T_{\text {hold }}=216 \mathrm{~ms}$. Shortly after the quench, the system remains in the unmagnetized $\left|m_{z}=0\right\rangle$ state, showing neither short-range spatial nor temporal variation (i.e. between frames). In contrast, condensates at longer times are spatially inhomogeneous and display spontaneous Larmor precession as indicated by the cyclical variation of signal strength vs. frame number. Orientations of axes and of the magnetic field are shown at left.

where $\tilde{n}$ is the column density of the gas, $\sigma=3 \lambda^{2} / 2 \pi$ is the resonant cross section, $\lambda=795 \mathrm{~nm}$ is the wavelength of the probe light, $\delta$ is the detuning from resonance, and $\gamma$ is the natural linewidth (Supplementary Note 2). Thus, given the column density of the gas and ignoring the small $\left\langle F_{y}^{2}\right\rangle$ signal, the phase-contrast signal is an instantaneous measure of one component, $F_{y}=\left\langle\hat{F}_{y}\right\rangle$, of the (dimensionless) magnetization of the gas.

We determined all three components of the vector magnetization density with repeated images of the same atomic sample. First, a sequence of 24 images was taken for which the magnetic field remained oriented along the $\hat{z}$ direction (Supplementary Note 2). Larmor precession of the transverse magnetization at around $35 \mathrm{kHz}$ is imaged as an aliased low-frequency oscillation (Fig. 1). The complex transverse magnetization $F_{T}=F_{x}+i F_{y}$ can then be determined from the relation $A(\vec{\rho}) \exp (i \phi(\vec{\rho}))=$ $i(5 / 6) \zeta(\vec{\rho}) F_{T}(\vec{\rho})$ where $A(\vec{\rho})$ and $\phi(\vec{\rho})$ are the amplitude and phase of this oscillation at each pixel position $\vec{\rho}$. The normalization constant $\zeta(\vec{\rho})$ was obtained by averaging the signal over all image frames and fitting with a Thomas-Fermi distribution for the density of the condensate. Following this sequence, two additional image frames were obtained within $5 \mathrm{~ms}$ in which the magnetic field was adiabatically reoriented in the $\hat{y}$ and $-\hat{y}$ directions. The longitudinal magnetization was determined from the difference between these last frames.

As shown in Fig. 1, at short times after the quench $\left(T_{\text {hold }}<50 \mathrm{~ms}\right)$, the transverse magnetization images show no significant variation across the cloud or between frames (similarly for the longitudinal magnetization images, which are not shown). This indicates that during this latency stage, the BEC remained in the unmagnetized scalar phase (the $\left|m_{z}=0\right\rangle$ state). Any magnetiza-

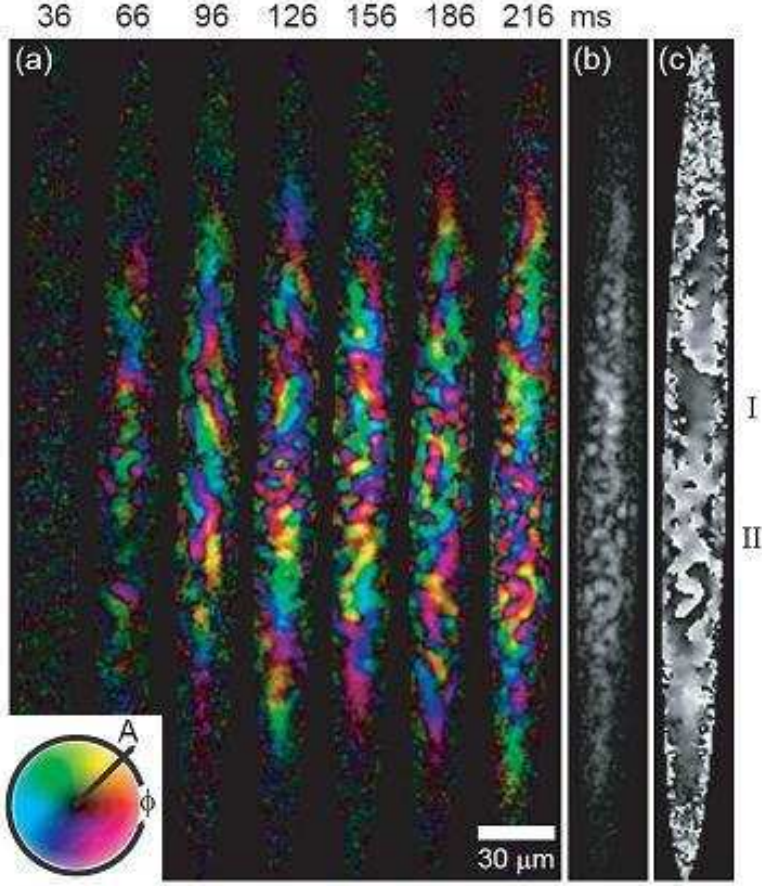

FIG. 2: In-situ images of ferromagnetic domains and domain walls. (a) The transverse magnetization density, measured for condensates at variable times $T_{\text {hold }}$ (indicated on top), is shown with the magnetization orientation $\left(\phi=\arg \left(F_{T}\right)\right)$ indicated by hue, and magnitude $\left(A=\zeta\left|F_{T}\right|\right)$ indicated by brightness. The maximum brightness, indicated by the color wheel at left, corresponds to full magnetization of the condensate center. For the data at $T_{\text {hold }}=216 \mathrm{~ms}$, the magnetization density (b) and orientation (c) are shown separately, and regions with either a large (I) or several small (II) magnetized domains are indicated. The gray scale in (c) covers the range 0 to $2 \pi$. Regions outside the condensate are indicated in black. Higher resolution picture available at http://physics.berkeley.edu/research/ultracold/pubs.html

tion during this stage either was too low in magnitude or varied over too short a length scale to be discerned by our imaging. At later times, a non-zero transverse magnetization signal spontaneously developed, yielding a Larmor precession signal that varied both in amplitude and in phase across the condensate. This observation indicates the spontaneous, spatially inhomogeneous breaking of $O(2)$ symmetry in the transverse plane in a direction given by the phase of Larmor precession.

In Fig. 2, the derived transverse magnetization for samples at variable times $T_{\text {hold }}$ are presented, rendered as color images to portray both the magnetization orientation (as hue) and amplitude (as brightness). These spatial maps show ferromagnetic domains of variable size and orientation arising spontaneously following the quench. The landscape of domains includes small regions of homogeneous magnetization, with bands of unmagnetized gas, i.e. domain walls, separating regions of nearly opposite orientation. One also observes larger ferromagnetic spin textures free of domain walls in which 
the magnetization orientation varies smoothly over tens of microns.

Ferromagnetic domains: The evolution toward ferromagnetism from the $\left|m_{z}=0\right\rangle$ state under the conditions $q<\left|c_{2}\right| n$ is a form of dynamical instability in a spinor BEC [12, 13, 14, 15]. This instability is a consequence of coherent collisional spin mixing in which pairs of atoms in the $\left|m_{z}=0\right\rangle$ state collide inelastically to produce atoms in the $\left|m_{z}= \pm 1\right\rangle$ states 3, 4, 9, 16]. Indeed, in our work, measurements of populations in the different magnetic sublevels through Stern-Gerlach analysis showed significant mixing of populations coincident with the onset of spontaneous Larmor precession.

One may also regard this instability as the phase separation of a two-component condensate [17, 18], recalling that the $\left|m_{z}=0\right\rangle$ state represents an equal superposition of the $\left|m_{\phi}= \pm 1\right\rangle$ eigenstates of any transverse spin operator, $\hat{F}_{\phi}=\hat{F}_{x} \cos \phi+\hat{F}_{y} \sin \phi$. Additionally, since $c_{2}<0$, the \pm 1 eigenstates of any spin component are immiscible [19]. Ferromagnetism thus arises by the spinodal decomposition of a binary gaseous mixture into neighboring regions of oppositely oriented transverse magnetization. Applying results from the two-component case, this phase separation is dominated by an instability with a characteristic exponential timescale $\tau_{F M}=\hbar / \sqrt{2\left|c_{2}\right| n}$ with $n$ being the total gas density. The wavevector of the dominant instability $k_{F M}=\sqrt{2 m\left|c_{2}\right| n} / \hbar$, defines the typical size $l=\pi k_{F M}^{-1}$ of single-component domains in the phase-separated fluid, and also the width $b \simeq k_{F M}^{-1}$ of domain walls in which the two components still overlap [20, 21].

To relate these predictions to our data, we considered the density-weighted transverse magnetization correlation function

$$
G_{T}(\delta \vec{\rho})=\operatorname{Re}\left[\frac{\sum_{\vec{\rho}}\left(\zeta(\vec{\rho}) F_{T}(\vec{\rho})\right)^{*}\left(\zeta(\vec{\rho}+\delta \vec{\rho}) F_{T}(\vec{\rho}+\delta \vec{\rho})\right)}{\sum_{\vec{\rho}} \zeta(\vec{\rho}) \zeta(\vec{\rho}+\delta \vec{\rho})}\right]
$$

At zero range, $G_{T}(0)$ measures the degree to which the condensate has evolved toward the ferromagnetic state. As shown in Fig. 3 (a), $G_{T}(0)$ rises after the quench from a near-zero background level characteristic of the unmagnetized initial state of the condensate to a nearly constant value of $G_{T}(0) \simeq 0.5$ during the ferromagnetic stage. This saturation value measures the area occupied by domain walls. Fitting $G_{T}(0)$ at early times $\left(T_{\text {hold }}<90 \mathrm{~ms}\right)$ to a rising exponential yields a time constant of $\tau=15(4) \mathrm{ms}$. This value is in agreement with the predicted $\tau_{F M}=13.7(3) \mathrm{ms}$, calculated using the peak density averaged over the $\hat{y}$ direction to account for its reduced dimensionality. Over the same period of evolution, no significant longitudinal magnetization was observed, confirming the presence of purely transverse ferromagnetic domains.

Spatial correlations in the transverse magnetization (Fig. 3(b)) are typified by a central region of positive correlations (near $\delta \vec{\rho}=0$ ) and then several equallysized regions of alternating negative and positive corre-
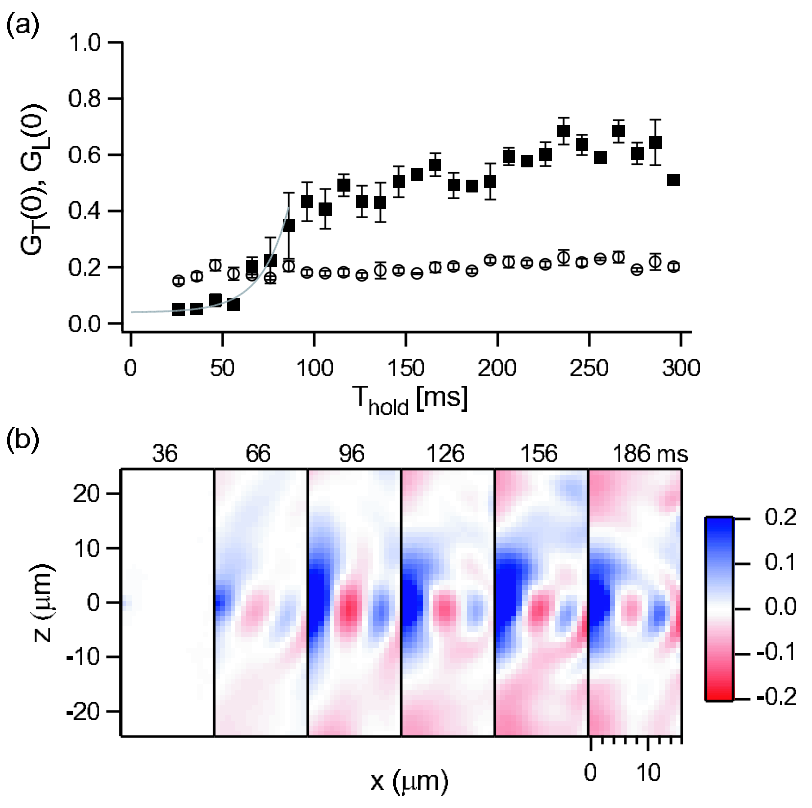

FIG. 3: Temporal and spatial evolution of ferromagnetism in a quenched spinor BEC. (a) The mean local squared transverse $\left(G_{T}(0)\right.$, squares) and longitudinal $\left(G_{L}(0)\right.$, circles) magnetization, averaged over several experimental repetitions (error bars indicate shot-to-shot rms fluctuations), is shown. $G_{T}(0)$ rises exponentially with time constant $\tau=15(4) \mathrm{ms}$ as determined by a fit to data for $T_{\text {hold }}<90 \mathrm{~ms}$ (gray line), and saturates at $G_{T}(0) \simeq 0.5$ at later times. No significant longitudinal magnetization is observed. The minimum values for $G_{T}(0)$ and $G_{L}(0)$, evident in the data for earliest $T_{\text {hold }}$, reflect residual noise due to processing either 24 or 2 frames, respectively, to obtain the magnetization density. (b) Maps of spatial correlations in the transverse magnetization $\left(G_{T}(x, z)\right)$, at variable $T_{\text {hold }}$ show alternating regions of positive and negative correlations in the narrow $\hat{x}$ direction, indicating that phase separation occurs primarily along $\hat{x}$ via a few discrete dynamical instability modes. At each $T_{\text {hold }}$, $G_{T}(x, z)$ was determined for ten repetitions of the experiment and then averaged.

lations displaced from one another in the narrow $(\hat{x})$ dimension of the condensate. We estimate a typical size for single-component domains as $\simeq 10 \mu \mathrm{m}$, twice the displacement at which the transverse spin-spin correlations changes sign, in good agreement with the predicted $\pi k_{F M}^{-1}=8.3(2) \mu \mathrm{m}$. The presence of negative correlation regions supports the model of spin-conserving phaseseparation discussed above. The preferential phase separation in the narrow $\hat{x}$ direction rather than along $\hat{z}$ is due to the broader momentum distribution of the unmagnetized condensate in that direction due to the finite condensate size. Thus, upon quenching the system, a greater population of atoms is available to seed the fastergrowing, shorter-wavelength instabilities with wavevector in the $\hat{x}$ direction. The presence of several alternations of positive and negative correlations further suggests that the phase separation occurs through a small number of discrete, unstable magnetization modes. 
Topological defects: The spontaneous symmetry breaking observed in this work is one of many examples of symmetry breaking that occur in nature. Symmetry breaking is presumed to have occurred at thermal phase transitions in the early universe, giving rise to the specific elementary particles and interactions observed in the present day. An important aspect of rapid spontaneous symmetry breaking, whether in the laboratory or of a cosmological nature, is the creation of topological defects, a process described theoretically by Kibble 22] and Zurek 23]. The types of topological defects that may be formed depend on the group structure of the ground-state manifold reached at the transition.

Analogues of cosmological topological defect formation have been studied using liquid crystals 24] and superfluid helium [25, 26, 27]. In comparison with these previous experiments, the present investigation focuses on a simpler physical system, in which the time for thermal equilibration and symmetry breaking is much longer than that needed to bring the system across the symmetry-breaking transition. Furthermore, the present work focuses on a quantum rather than a thermal phase transition.

In our two-dimensional system, spin-vortices are topological point defects about which the orientation of magnetization has a $2 \pi n$ winding with $n$ being a non-zero integer. Following a procedure for identifying such defects in our data (Supplementary Note 3), spin-vortex defects were observed with high confidence in about one third of all images containing significant ferromagnetism ( $T_{\text {hold }}>90 \mathrm{~ms}$ ), with some images containing up to four vortices. Data revealing one such spin-vortex are shown in Fig. 4. For this vortex, the central region of near-zero Larmor precession amplitude has a diameter of about 3 $\mu \mathrm{m}$, comparable to the spin healing length $\xi_{s}=2.4 \mu \mathrm{m}$ defined earlier. All the vortices we observed were singly quantized, with no apparent preferred direction of circulation.

Many types of vortices that can occur in a gas with a multi-component order parameter can be distinguished by the composition of their cores [28]. Based on our measurements of the transverse and longitudinal magnetization at the vortex core, the spin-vortices seen in our experiment appear to have unmagnetized filled cores. This observation supports their characterization as "polarcore" spin-vortices (denoted as $(1,0,-1)$ vortices in Ref. [28]), for which the superfluid order parameter is a superposition of atoms in the $\left|m_{z}=1\right\rangle$ state rotating with one quantum of circulation, atoms in the $\left|m_{z}=-1\right\rangle$ state rotating with one quantum of circulation in the opposite sense, and non-rotating atoms in the unmagnetized $\left|m_{z}=0\right\rangle$ state, which also fill the vortex core. Such a vortex is thus characterized by zero net mass circulation and a spin current with one quantum of circulation. The origin for such spin currents is presumably the spinodal decomposition by which ferromagnetism emerges from the unmagnetized cloud. The alternate identification of these vortices as merons [29, 30] is ruled out by the absence of a longitudinal magnetization signal at the vortex core.

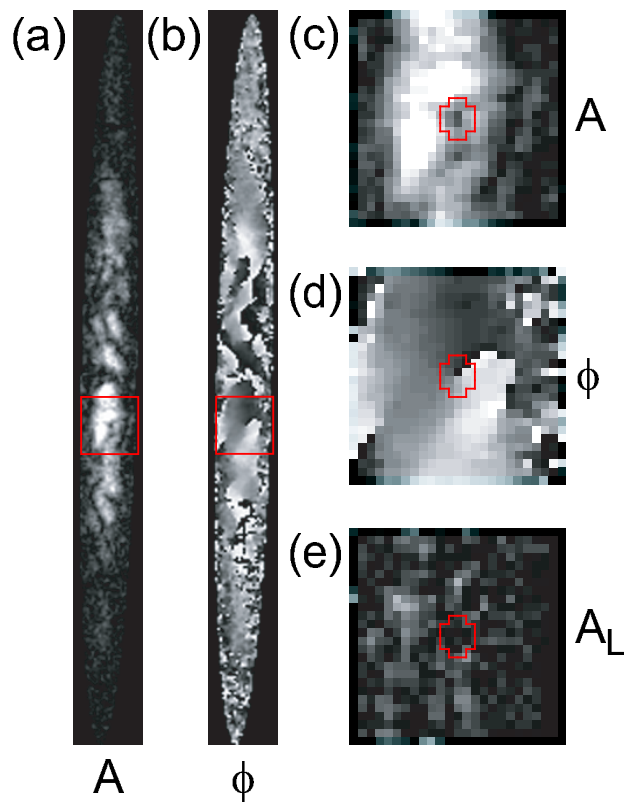

FIG. 4: In-situ detection of a polar core spin-vortex. Spatial maps of the transverse magnetization (a) magnitude $\left(A=\zeta\left|F_{T}\right|\right)$ and $(\mathrm{b})$ orientation $\left(\phi=\arg \left(F_{T}\right)\right)$ are shown for a sample imaged at $T_{\text {hold }}=150 \mathrm{~ms}$. Data from a portion of the condensate, indicated by boxes, are magnified, showing the transverse magnetization (c) magnitude and (d) orientation and also (e) the magnitude of the longitudinal magnetization $\left(A_{L}=\zeta\left|F_{z}\right|\right)$. The phase along a closed path (indicated in red), surrounding a region of near-zero transverse magnetization and satisfying the criteria stated in the text, shows a net winding of $2 \pi$, revealing the presence of a spin-vortex defect with its core within the closed path. The core shows no significant longitudinal magnetization, allowing the identification of the defect as a polar core spin-vortex. The gray scale for images $(\mathrm{a}, \mathrm{c}, \mathrm{e})$ is the same. The field of view is $31 \times$ $320 \mu \mathrm{m}$ for images (a,b), and $26 \times 26 \mu \mathrm{m}$ for images (c-e).

In contrast with their topological nature in some other magnetic systems, domain walls in a $F=1$ ferromagnet are not topologically stable; rather, they may decay by the formation of spin vortex-antivortex pairs. As discussed above, we observe domain walls that form at the onset of visible ferromagnetism in the gas, permeate the inhomogeneous clouds, and persist for all times thereafter. The persistence of domain walls in our system is a subject for future investigation.

Of keen interest for future studies is the distinction between thermal and quantum fluctuations as seeds for the unstable symmetry-breaking process. In this work, the forced depletion of atoms not in the $\left|m_{z}=0\right\rangle$ state suggests that ferromagnetism formed purely by the amplification of quantum fluctuations, i.e. shot noise, a suggestion that warrants experimental justification. Timeresolved experiments in which one varies the rate at which the system is swept into the ferromagnetic state may also uncover universal temporal dynamics that typify this and other quantum phase transitions. Our method of nondestructive magnetization imaging also al- 
lows the behavior of a single quenched condensate to be studied in detail, permitting an examination of the dynamical evolution of domain walls and spin-vortices.

We thank E. Mueller, J. Moore, and A. Vishwanath for helpful comments, J. Guzman for experimental assistance, and the NSF and David and Lucile Packard Foundation for financial support. S.R.L. acknowledges support from the NSERC.

Supplementary Note 1: Experimental sequence Optically-trapped BECs used for this work were obtained by loading around $10^{8}$ atoms in the $\left|F=1, m_{z}=-1\right\rangle$ state with temperature of $2.5 \mu \mathrm{K}$ into an optical dipole trap (ODT). The ODT was formed by a single focus laser beam with a wavelength of $825 \mathrm{~nm}$ that is linearly polarized to ensure that all components experience the same trap potential. The weak quadratic dependance of the trap depth on $m_{F}$ is negligible. After loading the atoms, all were placed in the $\left|m_{z}=0\right\rangle$ magnetic sublevel using rf rapid adiabatic passage followed by application of a transient magnetic field gradient of $4 \mathrm{G} / \mathrm{cm}$. Holding the magnetic field at a magnitude of $2 \mathrm{G}$, the optical trapping beam was decreased in power over $400 \mathrm{~ms}$, lowering the trap depth to $k_{B} \times 350 \mathrm{nK}$ and the temperature of the gas to $\sim 40 \mathrm{nK}$, well below the Bose-Einstein condensation temperature. This yielded nearly-pure condensates of $2.1(1) \times 10^{6}$ atoms.

After forming the condensate, the magnetic field was ramped within $5 \mathrm{~ms}$ to a value of $50 \mathrm{mG}$ oriented in the $\hat{z}$ direction. The field was held at this setting for a variable time before the imaging sequence. Field gradients along the $\hat{x}$ and $\hat{z}$ directions were nulled to less than 0.2 $\mathrm{mG} / \mathrm{cm}$. A Stern-Gerlach analysis of populations in each of the magnetic sublevels was applied to establish that the field ramp was sufficiently slow as not to alter the spin state of the condensate. Such an analysis bounded the populations in each of the $\left|m_{z}= \pm 1\right\rangle$ spin states to be less than $0.3 \%$ of the total population, both before and right after the field ramp.

Supplementary Note 2: Magnetization imaging The probe light for the phase contrast images was derived from a diode laser detuned $\delta=-200 \mathrm{MHz}$ from the $5 S_{1 / 2}(F=1) \rightarrow 5 P_{1 / 2}\left(F^{\prime}=2\right)$ transition in ${ }^{87} \mathrm{Rb}$. The imaging sequence to determine the transverse magnetization consisted of a sequence of 24 frames for which the probe was pulsed at a strobe frequency of around $10 \mathrm{kHz}$. The duration of each individual pulse was around $1 \mu \mathrm{s}$.

Supplementary Note 3: Image Analysis The determination of spin vortices in the magnetization images relied on the identification of a core of "dark" pixels, consistent with zero Larmor precession amplitude, which were surrounded by "bright" pixels with a finite amplitude and well-defined phase of Larmor precession. We identify vortices based on two criteria: (1) that there is an island of dark pixels, a candidate for the vortex core, which is surrounded entirely by bright pixels and that is at least two bright pixels away from nearby dark pixels, and (2) that the transverse magnetization traced along a closed loop through bright pixels surrounding the core have a non-zero net winding. The distinction between bright and dark pixels (at about one quarter of the maximum Larmor precession amplitude) was chosen so as to eliminate false-positive vortex detections in simulated data taking into account the measured noise and resolution of our imaging system, and using estimates for the width of contiguous domain walls.
[1] Stenger, J. et al. Nature 396, 345 (1998).

[2] Görlitz, A. et al. Phys. Rev. Lett. 90, 090401 (2003).

[3] Chang, M.-S. et al. Phys. Rev. Lett. 92, 140403 (2004).

[4] Schmaljohann, H. et al. Phys. Rev. Lett. 92, 040402 (2004).

[5] Two-component gases of ${ }^{87} \mathrm{Rb}$, in which the internal states are treated analogously to those of a spin- $1 / 2$ magnetic dipole, have also been studied; see Hall, D.S., Matthews, M.R., Wieman, C.E., and Cornell, E.A. Measurements of relative phase in two-component BoseEinstein condensates. Phys. Rev. Lett. 81, 1543 (1998).

[6] Ho, T.-L. Phys. Rev. Lett. 81, 742 (1998).

[7] Ohmi, T. and Machida, K. J. Phys. Soc. Jpn. 67, 1822 (1998).

[8] Klausen, N. N., Bohn, J. L., and Greene, C. H. Phys. Rev. A 64, 053602 (2001).

[9] Chang, M.-S. et al. Nature Physics 1, 111 (2005).

[10] The linear Zeeman effect may be neglected due to conservation of magnetization in our system.

[11] Higbie, J. M. et al. Phys. Rev. Lett. 95, 050401 (2005).

[12] Pu, H. et al. Phys. Rev. A 60, 1463 (1999).

[13] Robins, N. P., Zhang, W., Ostrovskaya, E. A., and Kivshar, Y. S. Phys. Rev. A 64, 021601(R) (2001).
[14] Saido, H. and Ueda, M. Phys. Rev. A 72, 023610 (2005).

[15] Zhang, W. et al. Phys. Rev. Lett. 95, 180403 (2005).

[16] Widera, A. et al. Phys. Rev. Lett. 95, 190405 (2005).

[17] Hall, D. S. et al. Phys. Rev. Lett. 81, 4531 (1998).

[18] Miesner, H.-J. et al. Phys. Rev. Lett. 82, 2228 (1999).

[19] Stamper-Kurn, D. M. and Ketterle, W. In Coherent Matter Waves, Kaiser, R., Westbrook, C., and David, F., editors, 137 - 218. Springer-Verlag, New York (2001).

[20] Goldstein, E. V. and Meystre, P. Phys. Rev. A 55, 2935 (1997).

[21] Timmermans, E. Phys. Rev. Lett. 81, 5718 (1997).

[22] Kibble, T. W. B. Journal of Physics A: Mathematics and General 9, 1387 (1976).

[23] Zurek, W. H. Nature 317, 505 (1985).

[24] Chuang, I., Durrer, R., Turok, N., and Yurke, B. Science 251, 1336 (1991).

[25] Hendry, P. C. et al. Nature 368, 315 (1994).

[26] Ruutu, V. M. H. et al. Nature 382, 334 (1996).

[27] Bauerle, C. et al. Nature 382, 332 (1996).

[28] Isoshima, T., Machida, K., and Ohmi, T. J. Phys. Soc. Jpn. 70, 1604 (2001).

[29] Mermin, N. D. and Ho, T.-L. Phys. Rev. Lett. 36, 594 (1976). 
[30] Saito, H., Kawaguchi, Y., and Ueda, M. Preprint, arXiv:cond-mat/0512216 